\title{
LEFT and RIGHT HANDEDNESS of FERMIONS and BOSONS
}

\author{
NORMA MANKOČ BORŠTNIK \\ Dept. of Physics, University of Ljubljana, Jadranska 19, and \\ J. Stefan Institute, Jamova 39, Ljubljana, 1111, Slovenia \\ and \\ ANAMARIJA BORŠTNIK \\ Department of Physics, University of Ljubljana, Jadranska 19
}

\begin{abstract}
It is shown, by using Grassmann space to describe the internal degrees of freedom of fermions and bosons, that the Weyl like equation exists not only for massless fermions but also for massless gauge bosons. The corresponding states have well defined helicity and handedness. It is also shown that spinors and gauge bosons only interact if they are of the same handedness.
\end{abstract}

PACS: 04.50.+h, 11.10.Kk,11.30.-j,12.10.-g

\section{Introduction.}

We discuss in this paper the properties of massless fermions and gauge bosons, described by the irreducible representations of the Poincaré group. We show that massless spinor and gauge vector representations exist, induced from those of a little group, which are eigenstates of the helicity $h=\frac{\vec{M} \cdot \vec{p}}{|\vec{M} \cdot \vec{p}|}$ and the handednes: $\Gamma$ operator, with $M_{i}=\frac{1}{2} \epsilon_{i j k} M^{j k} ; i, j, k \in\{1,2,3\},\left(M^{a b}\right.$ are the generators of the Lorentz group $S O(1,3)$, $p_{a}$ is the four momentum operator and $\Gamma=\frac{-i}{3 !} \eta \epsilon_{a b c d} M^{a b} M^{c d}$, with $\eta=1$ for fermions and $\eta=\frac{3}{8}$ for bosons, is one of the two invariants of the Lorentz group $S O(1,3)$ with $\epsilon_{a b c d \ldots}$. defined in Eq.(2.7)). Using Grassmann space to define the internal degrees of freedom, we show that both, spinors and gauge vectors, fulfil the operator equation

$$
\vec{M} \cdot \vec{p}=\xi p_{0} \Gamma
$$

with $\xi=\frac{1}{2}$ for spinors and $\xi=1$ for gauge bosons. The square of Eq.(1.1) leads to the equation $p^{2}=0$; for spinors this is true on the operator level, for gauge vectors only on the representation level.

*In the literature helicity and handedness are often mixed. Neutrino and antineutrino, for example, both left handed with $\Gamma=-1$, have helicity $h=-1$ and $h=+1$, respectively. 
We further show that the left handed fermions only interact with the left handed bosons and the right handed fermions only interact with the right handed bosons.

However, the left-right symmetry is not observed in nature. The photon and the gluon have well defined internal parity rather than handedness. They can accordingly be described by the sum of states with well defined handedness, which still have well defined helicity $h$ but they no longer fulfil the Weyl like equation (1.1). Massless fields have, no matter whether their spin is $\frac{1}{2}$ or 1 , two dimensional representations of the Lorentz group [1, 2, 3]. Because of that the gauge bosons (which are the three rather than the four vectors [4, 5, 6, 3, 7, 9, 10]) manifest the gauge invariance, generated by the little group generators [5, 7, 3, 8].

We discuss in this paper those properties of massless fermions and gauge bosons, which are connected with only spin degrees of freedom and are determined by the generators of the Lorentz transformations, the homogeneous and the discrete, of the group $S O(1,3)$ embedded into the group $S O(1,4)$ and are part of the Poincaré group.

To understand why the left-right symmetry is the broken symmetry in nature, one has to study not only spins but also charges: The authors of refs. [12, 13] restore the left-right symmetry in a phenomenological way, they label groups, introduced to describe charges, by the handedness: Their two weak charge $S U(2)$ groups carry representations of the left and right handedness, respectively, while the hypercharge group $U(1)$ and the $S U(3)$ colour charge group carry the representations of the left plus right symmetry. We shall comment on this point in the last section. We believe that the break of the leftright symmetry can only be understood within the unified theory of spins and charges. For this purpose the approach was introduced[10, 11], which describes all the internal degrees of freedom of fermions and bosons in an unique way. The approach assumes that space has d ordinary commuting and d Grassmann anticommuting coordinates, with $d \geq 15$. Since Grassmann space enables to define two kinds of generators of the Lorentz transformations, one of spinorial the other of vectorial character, two different kinds of representations exist, appropriate to describe spins and charges of fermions and gauge bosons, respectively [9, 10, 11]. For $d=15$, contain the representations, if determined by the operators forming the subgroups $S O(1,4), S U(3), S U(2), U(1)$ of the group $S O(1,14)$, all the vectors needed to describe spins and charges of all known fermions and gauge bosons, as well as the scalars [9]. Spins and charges are in the proposed theory unified, separately for spinors and separately for vectors.

Although many of the properties presented in this paper can well be understood in the usual group theoretical approach, the Grassmann space approach, with generators of groups represented by the differential operators in Grassmann space 10, 9, 11], offers the transparent way of understanding the behaviour of particles with respect to the internal degrees of freedom.

\footnotetext{
${ }^{\dagger}$ The standard electroweak model postulates in order to be in agreement with the experimental data that only left handed fermions carry the weak charge, while the right handed fermions do not. Accordingly right handed fermions do not interact with the weak bosons.
} 


\section{Coordinate Grassmann space, linear operators, Lorentz group $\mathrm{SO}(1,3)$ and representations.}

In this section we present the representations of the Lorentz group for spinors and vectors as polynomials in Grassmann space. We find spinors to form four times the two Weyl spinors, one of the left $(\Gamma=-1)$ (the $\left(\frac{1}{2}, 0\right)$ representation), the other of the right $(\Gamma=1) \quad$ ( the $\left(0, \frac{1}{2}\right)$ representation) handedness. The matrix representations for the generators of the Lorentz group and the invariants of the group are the same for all the four cases (Eq.2.11a). We find vectors to form a true scalar $(\Gamma=0)$ and a pseudo scalar $(\Gamma=0)$ representation with zero handedness, the three left handed vectors $(\Gamma=-1)$ ) ( the $(1,0)$ representation), the three right handed vectors $(\Gamma=1)$ ( the $(0,1)$ representation), and the two four vectors of zero handedness $(\Gamma=0)$ (the $\left(\frac{1}{2}, \frac{1}{2}\right)$ representations).

We first briefly repeat a few definitions concerning d-dimensional Grassmann space, linear Grassmann space spanned over the space of anticommuting coordinates, linear operators defined in this space and the Lie algebra of generators of the Lorentz transformations [9, 10, 11, 14]. We solve the eigenvalue problem and present the representations.

\subsection{Coordinate space with Grassmann character.}

We define a d-dimensional Grassmann space of real anticommuting coordinates $\left\{\theta^{a}\right\}$, $a=0,1,2,3,5,6, \ldots, d$, satisfying the anticommutation relations $\theta^{a} \theta^{b}+\theta^{b} \theta^{a}:=\left\{\theta^{a}, \theta^{b}\right\}=0$, called the Grassmann algebra [9, 11, 14]. The metric tensor $\eta_{a b}=\operatorname{diag}(1,-1,-1$, $-1, \ldots,-1)$ lowers the indices of a vector $\left\{\theta^{a}\right\}=\left\{\theta^{0}, \theta^{1}, \ldots, \theta^{d}\right\}, \theta_{a}=\eta_{a b} \theta^{b}$. Linear transformation actions on vectors $\left(\alpha \theta^{a}+\beta x^{a}\right), \quad\left(\alpha \hat{\theta}^{a}+\beta \dot{x}^{a}\right)=L_{b}^{a}\left(\alpha \theta^{b}+\beta x^{b}\right)$, which leave forms $\left(\alpha \theta^{a}+\beta x^{a}\right)\left(\alpha \theta^{b}+\beta x^{b}\right) \eta_{a b}$ invariant, are called the Lorentz transformations. Here $\left(\alpha \theta^{a}+\beta x^{a}\right)$ is a vector of $\mathrm{d}$ anticommuting components and $\mathrm{d}$ commuting $\left(x^{a} x^{b}-x^{b} x^{a}=0\right)$ components, and $\alpha$ and $\beta$ are two complex numbers. The requirement that forms $\left(\alpha \theta^{a}+\beta x^{a}\right)\left(\alpha \theta^{b}+\beta x^{b}\right) \eta_{a b}$ are scalars with respect to the above linear transformations, leads to the equations $L^{a}{ }_{c} L^{b}{ }_{d} \eta_{a b}=\eta_{c d}$.

\section{$2.2 \quad$ Linear vector space.}

A linear space spanned over a Grassmann coordinate space of $\mathrm{d}$ coordinates has the dimension $2^{d}$. If monomials $\theta^{\alpha_{1}} \theta^{\alpha_{2}} \ldots \theta^{\alpha_{n}}$ are taken as a set of basic vectors with $\alpha_{i} \neq \alpha_{j}$, half of the vectors have an even (those with an even $n$ ) and half of the vectors have an odd (those with an odd n) Grassmann character. Any vector in this space may be represented as a linear superposition of monomials

$$
f(\theta)=\alpha_{0}+\sum_{i=1}^{d} \alpha_{a_{1} a_{2} . . a_{i}} \theta^{a_{1}} \theta^{a_{2}} \ldots . \theta^{a_{i}}, \quad a_{k}<a_{k+1},
$$

where constants $\alpha_{0}, \alpha_{a_{1} a_{2} . . a_{i}}$ are complex numbers. 


\subsection{Linear operators.}

In Grassmann space the left derivatives have to be distinguished from the right derivatives, due to the anticommuting nature of the coordinates 19, 11, 14]. We shall make use of left derivatives $\overrightarrow{\partial^{\theta}}{ }_{a}:=\frac{\vec{\partial}}{\partial \theta^{a}}, \quad \overrightarrow{\partial^{\theta} a}:=\eta^{a b} \vec{\partial}_{b}{ }_{b}$, on vectors of the linear space of monomials $f(\theta)$, defined as follows: $\overrightarrow{\partial^{\theta}}{ }_{a} \theta^{b} f(\theta)=\delta_{a}^{b} f(\theta)-\theta^{b} \vec{\partial}_{a}{ }_{a} f(\theta)$.

We define the linear operators [9, 11]

$$
p^{\theta a}:=i \overrightarrow{\partial^{\theta} a}, \quad \tilde{a}^{a}:=-i\left(p^{\theta a}+i \theta^{a}\right), \quad \tilde{\tilde{a}}^{a}:=\left(p^{\theta a}-i \theta^{a}\right) .
$$

According to the inner product defined in Eq.(2.8), the operators $\tilde{a}^{a}$ and $\tilde{\tilde{a}}^{a}$ are either hermitian or antihermitian operators $\quad \theta^{a+}=i \eta^{a a} p^{\theta a}, \quad p^{\theta a+}=-i \eta^{a a} p^{\theta a}, \quad \tilde{a}^{a+}=$ $\eta^{a a} \tilde{a}^{a}, \tilde{\tilde{a}}^{a+}=\eta^{a a} \tilde{\tilde{a}}^{a}$.

We define the generalized commutation relations (which follow from the corresponding Poisson brackets [9, 10, 11]):

$$
\{A, B\}:=A B-(-1)^{n_{A B}} B A,
$$

fulfilling the equation $\{A, B\}=(-1)^{n_{A B}+1}\{B, A\}$. Here $n_{A B}$ is defined as follows

$$
n_{A B}=\left\{\begin{array}{ll}
+1, & \text { if } A \text { and } B \text { have Grassmann odd character } \\
0, & \text { otherwise }
\end{array}\right\} .
$$

We find

$$
\left\{p^{\theta a}, p^{\theta b}\right\}=0=\left\{\theta^{a}, \theta^{b}\right\}, \quad\left\{p^{\theta a}, \theta^{b}\right\}=i \eta^{a b}, \quad\left\{\tilde{a}^{a}, \tilde{a}^{b}\right\}=2 \eta^{a b}=\left\{\tilde{\tilde{a}}^{a}, \tilde{\tilde{a}}^{b}\right\}, \quad\left\{\tilde{a}^{a}, \tilde{\tilde{a}}^{b}\right\}=0 .
$$

We see that $\theta^{a}$ and $p^{\theta a}$ form a Grassmann odd Heisenberg algebra, while $\tilde{a}^{a}$ and $\tilde{\tilde{a}}^{a}$ form the Clifford algebra. In this paper we shall make use of only $\tilde{a}^{a}$. We shall put $\tilde{\tilde{a}}^{a}$ equal zero. We shall comment on this point in Sect. 3 .

\subsection{Lie algebra of Lorentz group.}

We define two kinds of operators [9, 10]. The first ones are binomials of operators forming the Grassmann odd Heisenberg algebra

$$
S^{a b}:=\left(\theta^{a} p^{\theta b}-\theta^{b} p^{\theta a}\right) .
$$

The second ones are binomials of operators forming the Clifford algebra

$$
\tilde{S}^{a b}:=\frac{i}{4}\left[\tilde{a}^{a}, \tilde{a}^{b}\right],
$$

with $[A, B]:=A B-B A$. 
Either the operators $S^{a b}$ or $\tilde{S}^{a b}$ fulfil the Lie algebra of the Lorentz group $S O(1, d-1)$ in d-dimensional Grassmann space:

$$
\left\{M^{a b}, M^{c d}\right\}=i\left(M^{a d} \eta^{b c}+M^{b c} \eta^{a d}-M^{a c} \eta^{b d}-M^{b d} \eta^{a c}\right)
$$

with $M^{a b}$ equal either to $S^{a b}$ or to $\tilde{S}^{a b}$ and $M^{a b}=-M^{b a}$.

By solving the eigenvalue problem (see below) we find that operators $\tilde{S}^{a b}$ define the spinorial representations of the Lorentz group, while $S^{a b}$ define the vectorial representations of the Lorentz group $S O(1, d-1)$.

Group elements are in any of the two cases defined by: $\mathcal{U}(\omega)=e^{-\frac{i}{2} \omega_{a b} M^{a b}}$, where $\omega_{a b}$ are the parameters of the group.

It can be proved for any $\mathrm{d}$ that $M^{2}$ is the invariant of the Lorentz group $\left\{M^{2}, M^{c d}\right\}=$ $0, M^{2}=\frac{1}{2} M^{a b} M_{a b}$, and that for $\mathrm{d}=2 \mathrm{n}$ we can find an additional invariant $\Gamma$

$$
\Gamma=\eta \frac{i(-2 i)^{n}}{(2 n) !} \epsilon_{a_{1} a_{2} \ldots a_{2 n}} M^{a_{1} a_{2}} \ldots M^{a_{2 n-1} a_{2 n}}, \quad\left\{\Gamma, M^{c d}\right\}=0,
$$

where $\epsilon_{a_{1} a_{2} \ldots a_{2 n}}$ is the totally antisymmetric tensor with $2 n$ indices and with $\epsilon_{123 \ldots 2 n}=1$ and $\eta$ is a real constant. We choose $\eta=1$ for spinors and $\eta=\frac{3}{8}$ for vectors. This means that $M^{2}$ and $\Gamma$ are for $d=4$ the two invariants or Casimir operators of the group $S O(1,3)$.

While the invariant $M^{2}$ is trivial in the case when $M^{a b}$ has spinorial character, since $\left(\tilde{S}^{a b}\right)^{2}=\frac{1}{4} \eta^{a a} \eta^{b b}$ and $M^{2}$ is equal to a number $\frac{1}{2} \tilde{S}^{a b} \tilde{S}_{a b}=\frac{3}{2}$, it is a nontrivial differential operator in Grassmann space if $M^{a b}$ have vectorial character $\left(M^{a b}=S^{a b}\right)$. The invariant of Eq.(2.7) is always a nontrivial operator.

\subsection{Integrals on Grassmann space.}

We assume that differentials of Grassmann coordinates $d \theta^{a}$ fulfil the Grassmann anticommuting relations [9, 11, 14 $\left\{d \theta^{a}, d \theta^{b}\right\}=0$ and we introduce a single integral over the whole interval of $d \theta^{a}: \int d \theta^{a}=0, \quad \int d \theta^{a} \theta^{a}=1, \quad a=0,1,2,3,5, . ., d$, and the multiple integral over d coordinates $\int d^{d} \theta \theta^{0} \theta^{1} \theta^{2} \theta^{3} \theta^{4} \ldots \theta^{d}=1$, with $d^{d} \theta:=d \theta^{d} \ldots d \theta^{3} d \theta^{2} d \theta^{1} d \theta^{0}$ in the standard way.

We define [9, 11, 14] the inner product of two vectors $\langle\varphi \mid \theta\rangle$ and $\langle\theta \mid \chi\rangle$, with $\langle\varphi \mid \theta\rangle=$ $\langle\theta \mid \varphi\rangle^{*}$ as follows:

$$
\langle\varphi \mid \chi\rangle=\int d^{d} \theta(\omega\langle\varphi \mid \theta\rangle)\langle\theta \mid \chi\rangle,
$$

with the weight function $\omega=\prod_{k=0,1,2,3, . ., d}\left(\frac{\partial}{\partial \theta^{k}}+\theta^{k}\right)$, which operates on the first function $\langle\varphi \mid \theta\rangle$ only, and we define $\left(\alpha^{a_{1} a_{2} \ldots a_{k}} \theta^{a_{1}} \theta^{a_{2}} \ldots \theta^{a_{k}}\right)^{+}=\left(\theta^{a_{k}}\right) \ldots . .\left(\theta^{a_{2}}\right)\left(\theta^{a_{1}}\right)\left(\alpha^{a_{1} a_{2} \ldots a_{k}}\right)^{*}$.

According to the above definition of the inner product the generators of the Lorentz transformations (Eqs.(2.5b)) are self adjoint ( if $a \neq 0$ and $b \neq 0$ ) or antiself adjoint ( if $a=0$ or $b=0$ ) operators. 
The volume element $d^{d} \theta$ and the weight function $\omega$ are invariants with respect to the Lorentz transformations, both are scalar densities of weight -1 .

According to Eqs.(2.2) and (2.5) we find

$$
S^{a b}=i\left(\theta^{a} \frac{\partial}{\partial \theta_{b}}-\theta^{b} \frac{\partial}{\partial \theta_{a}}\right), \quad \tilde{a}^{a}=\left(\frac{\partial}{\partial \theta_{a}}+\theta^{a}\right), \quad \tilde{S}^{a b}=\frac{i}{2}\left(\frac{\partial}{\partial \theta_{a}}+\theta^{a}\right)\left(\frac{\partial}{\partial \theta_{b}}+\theta^{b}\right), \text { if } a \neq b .
$$

\subsection{Eigenvalue problem.}

To find eigenvectors of any operator $A$, we solve the eigenvalue problem

$$
\left\langle\theta\left|\tilde{A}_{i}\right| \tilde{\varphi}\right\rangle=\tilde{\alpha}_{i}\langle\theta \mid \tilde{\varphi}\rangle, \quad\left\langle\theta\left|A_{i}\right| \varphi\right\rangle=\alpha_{i}\langle\theta \mid \varphi\rangle, \quad i=\{1, p\}
$$

where $\tilde{A}_{i}$ and $A_{i}$ stand for $p$ commuting operators of spinorial and vectorial character, respectively and $\tilde{\alpha}_{i}$ and $\alpha_{i}$ for the corresponding eigenvalues.

To solve equations (2.10) we express the operators in the coordinate representation and write the eigenvectors as polynomials of $\theta^{a}$. We orthonormalize the vectors according to the inner product, defined in Eq.(2.8), $\left\langle\left.{ }^{\alpha} \tilde{\varphi}_{i}\right|^{\beta} \tilde{\varphi}_{j}\right\rangle=\delta^{\alpha \beta} \delta_{i j}, \quad\left\langle\left.{ }^{\alpha} \varphi_{i}\right|^{\beta} \varphi_{j}\right\rangle=\delta^{\alpha \beta} \delta_{i j}$, where index $\alpha$ distinguishes between vectors of different irreducible representations and index $\mathrm{j}$ between vectors of the same irreducible representation. This determines the orthonormalization condition for spinorial and vectorial representations, respectively.

\subsection{Representations of spinors and vectors of $S O(1,3)$ embedded into $S O(1,4)$.}

We solve the eigenvalue problem for the invariants $M^{2}$ and $\Gamma$ as well as for the commuting operators $M^{12}$ and $M^{03}$ of the Lorentz group $S O(1,3)$ for either spinorial or vectorial operators (Eqs.(2.9)). We take $d=5$ for the reason that the operator of an even Grassmann character $\tilde{\gamma}=-2 i \tilde{S}^{5 m}=\tilde{a}^{5} \tilde{a}^{m}, m \in\{0,1,2,3\}$ can be defined, with all the properties of the Dirac $\gamma^{m}$ operators $f$. In $d=5$ dimensional Grassmann space there are 16 vectors of an odd and 16 vectors of an even Grassmann character. We find within the odd vectors the irreducible representations of the Lorentz group $S O(1,3)$ for the spinorial operators and within the even vectors the irreducible representations of the Lorentz group $S O(1,3)$ for the vectorial operators

In the spinorial case we find four times two spinors, one left $(\Gamma=-1)$ and one right $(\Gamma=1)$ handed, each two spinor coupled to a bispinor by the operator $\tilde{\gamma}^{m}$. The four different two spinor representations distinguish among themselves with respect to the

\footnotetext{
$\ddagger$ Having an even Grassmann character $\tilde{\gamma}^{a}$ transform spinors into spinors, otherwise they would change the Grassmann character of fields, causing the supersymmetric transformations[9].

$\S$ The reason for such a choice is that in the canonical quantization of fields the Grassmann odd vectors quantize to fermions, the Grassmann even to bosons.
} 
discrete transformations of the Lorentz group $S O(1,3): \vec{\theta} \longrightarrow-\vec{\theta}, \theta^{0} \longrightarrow-\theta^{0}$ and the complex conjugation. We present these representations in Table I. We introduce the notation: $\tilde{S}_{i}=\frac{1}{2} \epsilon_{i j k} \tilde{S}^{j k}, \quad \tilde{K}_{i}=\tilde{S}^{0 i}$. In Eq.(2.11a) we present the matrix representation for the generators of the Lorentz transformations of the group $S O(1,3)$, for the invariant $\tilde{\Gamma}$ and for $\tilde{\gamma}^{0}$ for any of the four vectors. Starting from any of the sixteen vectors, all the others can be obtained by applying on the starting one either the generators $\tilde{S}^{m n}$, or $\tilde{\gamma}^{m}$ or the generators of the discrete symmetries: space inversion, time inversion or complex conjugation.

\begin{tabular}{|c|c||c||r|r|r|c|}
\hline$\alpha$ & $i$ & $\left\langle\left.\theta\right|^{\alpha} \tilde{\varphi}_{i}\right\rangle$ & $\tilde{S}^{3}$ & $\tilde{K}^{3}$ & $\tilde{\Gamma}$ & $\tilde{\mathcal{S}}^{2}$ \\
\hline \hline 1 & 1 & $-\frac{1}{2}\left(\theta^{1}+i \theta^{2}\right)\left(\theta^{0}+\theta^{3}\right) \theta^{5}$ & $\frac{1}{2}$ & $-\frac{i}{2}$ & -1 & $\frac{3}{2}$ \\
\hline 1 & 2 & $\frac{1}{2}\left(1-i \theta^{1} \theta^{2}\right)\left(1+\theta^{0} \theta^{3}\right) \theta^{5}$ & $-\frac{1}{2}$ & $\frac{i}{2}$ & -1 & $\frac{3}{2}$ \\
\hline 2 & 1 & $\frac{1}{2}\left(\theta^{1}+i \theta^{2}\right)\left(1+\theta^{0} \theta^{3}\right)$ & $\frac{1}{2}$ & $\frac{i}{2}$ & 1 & $\frac{3}{2}$ \\
\hline 2 & 2 & $-\frac{1}{2}\left(1-i \theta^{1} \theta^{2}\right)\left(\theta^{0}+\theta^{3}\right)$ & $-\frac{1}{2}$ & $-\frac{i}{2}$ & 1 & $\frac{3}{2}$ \\
\hline \hline 3 & 1 & $-\frac{1}{2}\left(\theta^{1}+i \theta^{2}\right)\left(1-\theta^{0} \theta^{3}\right)$ & $\frac{1}{2}$ & $-\frac{i}{2}$ & -1 & $\frac{3}{2}$ \\
\hline 3 & 2 & $\frac{1}{2}\left(1-i \theta^{1} \theta^{2}\right)\left(\theta^{0}-\theta^{3}\right)$ & $-\frac{1}{2}$ & $\frac{i}{2}$ & -1 & $\frac{3}{2}$ \\
\hline 4 & 1 & $\frac{1}{2}\left(\theta^{1}+i \theta^{2}\right)\left(\theta^{0}-\theta^{3}\right) \theta^{5}$ & $\frac{1}{2}$ & $\frac{i}{2}$ & 1 & $\frac{3}{2}$ \\
\hline 4 & 2 & $\frac{1}{2}\left(1-i \theta^{1} \theta^{2}\right)\left(1-\theta^{0} \theta^{3}\right) \theta^{5}$ & $-\frac{1}{2}$ & $-\frac{i}{2}$ & 1 & $\frac{3}{2}$ \\
\hline \hline 5 & 1 & $-\frac{1}{2}\left(1+i \theta^{1} \theta^{2}\right)\left(\theta^{0}+\theta^{3}\right)$ & $\frac{1}{2}$ & $-\frac{i}{2}$ & -1 & $\frac{3}{2}$ \\
\hline 5 & 2 & $\frac{1}{2}\left(\theta^{1}-i \theta^{2}\right)\left(1+\theta^{0} \theta^{3}\right)$ & $-\frac{1}{2}$ & $\frac{i}{2}$ & -1 & $\frac{3}{2}$ \\
\hline 6 & 1 & $-\frac{1}{2}\left(1+i \theta^{1} \theta^{2}\right)\left(1+\theta^{0} \theta^{3}\right) \theta^{5}$ & $\frac{1}{2}$ & $\frac{i}{2}$ & 1 & $\frac{3}{2}$ \\
\hline 6 & 2 & $\frac{1}{2}\left(\theta^{1}-i \theta^{2}\right)\left(\theta^{0}+\theta^{3}\right) \theta^{5}$ & $-\frac{1}{2}$ & $-\frac{i}{2}$ & 1 & $\frac{3}{2}$ \\
\hline \hline 7 & 1 & $-\frac{1}{2}\left(1+i \theta^{1} \theta^{2}\right)\left(1-\theta^{0} \theta^{3}\right) \theta^{5}$ & $\frac{1}{2}$ & $-\frac{i}{2}$ & -1 & $\frac{3}{2}$ \\
\hline 7 & 2 & $\frac{1}{2}\left(\theta^{1}-i \theta^{2}\right)\left(\theta^{0}-\theta^{3}\right) \theta^{5}$ & $-\frac{1}{2}$ & $\frac{i}{2}$ & -1 & $\frac{3}{2}$ \\
\hline 8 & 1 & $-\frac{1}{2}\left(1+i \theta^{1} \theta^{2}\right)\left(\theta^{0}-\theta^{3}\right)$ & $\frac{1}{2}$ & $\frac{i}{2}$ & 1 & $\frac{3}{2}$ \\
\hline 8 & 2 & $-\frac{1}{2}\left(\theta^{1}-i \theta^{2}\right)\left(1-\theta^{0} \theta^{3}\right)$ & $-\frac{1}{2}$ & $-\frac{i}{2}$ & 1 & $\frac{3}{2}$ \\
\hline
\end{tabular}

Table I. Irreducible representations of the Lorentz group $S O(1,3)$ embedded into the group $S O(1,4)$. The polynomials demonstrate the $S U(2) \times S U(2)$ structure of the Lorentz group $S O(1,3)$ and represent four times the left and the right handed Weyl spinor [9]. We use the notation $\tilde{\mathcal{S}}^{2}=\frac{1}{2} \tilde{S}^{a b} \tilde{S}_{a b}$.

$$
\begin{gathered}
\tilde{\Gamma}_{\alpha}=\left(\begin{array}{rr}
-I & 0 \\
0 & I
\end{array}\right), \quad \vec{S}_{\alpha}=\left(\begin{array}{rr}
\overrightarrow{\vec{s}} & 0 \\
0 & \overrightarrow{\vec{s}}
\end{array}\right), \quad \vec{K}_{\alpha}=\left(\begin{array}{rr}
-i \overrightarrow{\vec{s}} & 0 \\
0 & i \overrightarrow{\vec{s}}
\end{array}\right), \\
\tilde{\gamma}_{\alpha}^{0}=\left(\begin{array}{rr}
0 & I \\
I & 0
\end{array}\right), \quad \overrightarrow{\vec{s}}=\frac{1}{2} \vec{\sigma}, \quad I=\left(\begin{array}{ll}
1 & 0 \\
0 & 1
\end{array}\right), \\
\text { here } \vec{\sigma} \text { are Pauli matrices. One finds } \overrightarrow{\widetilde{K}}_{\alpha}=i \tilde{\Gamma}_{\alpha} \vec{S}_{\alpha} .
\end{gathered}
$$


In the vectorial case we find a true and a pseudo scalar of zero handedness, one three vector of the left handedness $(\Gamma=-1)$, one three vector of the right handedness $(\Gamma=1)$ and two four vectors of zero handedness. We present the results in Table II, using the equivalent notation as in the spinorial case. In Eq.(2.11b) we present the matrix representations of the operators for the two three vectors of different handedness. While the matrices are in the spinorial case of the dimension four times fourf are in the vectorial case of the dimension six times six.].

\begin{tabular}{|c|c||c||r|r|r|r|}
\hline$\alpha$ & $i$ & $\left\langle\left.\theta\right|^{\alpha} \varphi_{i}\right\rangle$ & $S^{3}$ & $K^{3}$ & $\Gamma$ & $\mathcal{S}^{2}$ \\
\hline \hline 1 & 1 & $\frac{1}{2}\left(\theta^{0}+\theta^{3}\right)\left(\theta^{1}+i \theta^{2}\right)$ & 1 & $-i$ & -1 & 4 \\
\hline 1 & 2 & $-\frac{1}{\sqrt{2}}\left(i \theta^{1} \theta^{2}-\theta^{0} \theta^{3}\right)$ & 0 & 0 & -1 & 4 \\
\hline 1 & 3 & $-\frac{1}{2}\left(\theta^{0}-\theta^{3}\right)\left(\theta^{1}-i \theta^{2}\right)$ & -1 & $i$ & -1 & 4 \\
\hline \hline 2 & 1 & $\frac{1}{2}\left(\theta^{0}-\theta^{3}\right)\left(\theta^{1}+i \theta^{2}\right)$ & 1 & $i$ & 1 & 4 \\
\hline 2 & 2 & $-\frac{1}{\sqrt{2}}\left(i \theta^{1} \theta^{2}+\theta^{0} \theta^{3}\right)$ & 0 & 0 & 1 & 4 \\
\hline 2 & 3 & $-\frac{1}{2}\left(\theta^{0}+\theta^{3}\right)\left(\theta^{1}-i \theta^{2}\right)$ & -1 & $-i$ & 1 & 4 \\
\hline \hline 3 & 1 & 1 & 0 & 0 & 0 & 0 \\
\hline 4 & 1 & $i \theta^{0} \theta^{1} \theta^{2} \theta^{3}$ & 0 & 0 & 0 & 0 \\
\hline \hline 5 & 1 & $\frac{1}{2}\left(1+\theta^{0} \theta^{3}\right)\left(\theta^{1}-i \theta^{2}\right) \theta^{5}$ & -1 & 0 & 0 & 3 \\
\hline 5 & 2 & $\frac{1}{2}\left(\theta^{0}-\theta^{3}\right)\left(1-i \theta^{1} \theta^{2}\right) \theta^{5}$ & 0 & $i$ & 0 & 3 \\
\hline 5 & 3 & $\frac{1}{2}\left(1-\theta^{0} \theta^{3}\right)\left(\theta^{1}+i \theta^{2}\right) \theta^{5}$ & 1 & 0 & 0 & 3 \\
\hline 5 & 4 & $\frac{1}{2}\left(\theta^{0}+\theta^{3}\right)\left(1+i \theta^{1} \theta^{2}\right) \theta^{5}$ & 0 & $-i$ & 0 & 3 \\
\hline \hline 6 & 1 & $\frac{1}{2}\left(1-\theta^{0} \theta^{3}\right)\left(\theta^{1}-i \theta^{2}\right) \theta^{5}$ & -1 & 0 & 0 & 3 \\
\hline 6 & 2 & $\frac{1}{2}\left(\theta^{0}-\theta^{3}\right)\left(1+i \theta^{1} \theta^{2}\right) \theta^{5}$ & 0 & $i$ & 0 & 3 \\
\hline 6 & 3 & $\frac{1}{2}\left(1+\theta^{0} \theta^{3}\right)\left(\theta^{1}+i \theta^{2}\right) \theta^{5}$ & 1 & 0 & 0 & 3 \\
\hline 6 & 4 & $\frac{1}{2}\left(\theta^{0}+\theta^{3}\right)\left(1-i \theta^{1} \theta^{2}\right) \theta^{5}$ & 0 & $-i$ & 0 & 3 \\
\hline
\end{tabular}

Table II. Irreducible representations of the Lorentz group $S O(1,3)$ embedded into the group $S O(1,4)$. The polynomials in Grassmann space represent the two scalars, the two three vectors and the two four vectors [9]. We use the notation $\mathcal{S}=\frac{1}{2} S^{a b} S_{a b}$.

$$
\Gamma=\left(\begin{array}{rr}
-I & 0 \\
0 & I
\end{array}\right), \quad \vec{S}=\left(\begin{array}{cc}
\vec{s} & 0 \\
0 & \vec{s}
\end{array}\right), \vec{K}=\left(\begin{array}{cc}
-i \vec{s} & 0 \\
0 & i \vec{s}
\end{array}\right),
$$

I The matrices are in the spinorial case of the dimension sixteen times sixteen, but having the block diagonal structure of dimension four times four, they are the direct sum of four times the four square matrices. In the vectorial case the corresponding sixteen times sixteen matrices are the direct sum of one six times six matrix, two one dimensional matrices and one eight times eight matrix.

$\|$ One can find also in the case of the two three vectors the matrices $\gamma^{0}$, which transform the left handed vectors into the right handed ones or opposite, but we don't find the operator realization of such matrices. 


$$
s_{1}=\frac{1}{\sqrt{2}}\left(\begin{array}{ccc}
0 & 1 & 0 \\
1 & 0 & 1 \\
0 & 1 & 0
\end{array}\right), s_{2}=\frac{1}{\sqrt{2}}\left(\begin{array}{rrr}
0 & -i & 0 \\
i & 0 & -i \\
0 & i & 0
\end{array}\right), \quad s_{3}=\left(\begin{array}{ccc}
1 & 0 & 0 \\
0 & 0 & 0 \\
0 & 0 & -1
\end{array}\right)
$$

It follows again that $\vec{K}=i \Gamma \vec{S}$.

\section{$3 \quad$ Particles in ordinary and Grassmann space.}

A particle which lives in a space of d ordinary commuting and d Grassmann anticommuting coordinates $X^{a}:=\left\{x^{a}, \theta^{a}\right\}$ moves along the supergeodesics 11. Choosing the appropriate action 11, 15] to describe the dynamics of such a particle, one derives the two Euler-Lagrange equations [1]]: $\frac{d p^{a}}{d \tau}=0, \quad \frac{d p^{\theta a}}{d \tau}=\frac{i}{2} m p^{a}$, and the three constraints:

$$
\chi^{1}:=p_{a} \tilde{a}^{a}=0, \quad \chi^{2}:=p^{a} p_{a}=0, \quad \chi_{a}^{3}:=\tilde{\tilde{a}}^{a}:=p^{\theta a}-i \theta^{a}=0, \quad \text { with } \tilde{a}^{a}:=-i p^{\theta a}+\theta^{a},
$$

where $p^{a}$ is the conjugate momentum to the coordinate $x^{a}, p^{\theta a}$ the conjugate momentum to the coordinate $\theta^{a}$ and $m$ is the parameter of the action. One also finds the generators of the Lorentz transformations:

$$
M^{a b}=L^{a b}+\tilde{S}^{a b}, L^{a b}=x^{a} p^{b}-x^{b} p^{a}, \tilde{S}^{a b}=\frac{i}{4}\left(\tilde{a}^{a} \tilde{a}^{b}-\tilde{a}^{b} \tilde{a}^{a}\right),
$$

demonstrating that parameters of the Lorentz transformations are the same in both spaces and that coordinates in Grassmann space are proportional to their conjugate momenta.

In the canonical quantization of coordinates [1], the momenta $p^{a}$ and $p^{\theta a}$ and accordingly quantities $\tilde{a}^{a}$ and $\tilde{\tilde{a}}^{a}$ become operators, fulfilling Eqs. (2.2,2.4) of Sect.2.3.. The two constraints of Eq.(3.1) lead to the Dirac and Klein-Gordon equations, provided that coordinates $\theta^{a}$ and their conjugate momenta $p^{\theta a}$, which appear in the equations of motion, are replaced by $\frac{1}{2} \tilde{a}^{a}$ and $\frac{1}{2 i} \tilde{a}^{a}$, respectively, while $\tilde{\tilde{a}}^{a}$ are put equal to zero, and that the generator of the Lorentz transformations $-2 i \tilde{S}^{5 m}, m=0,1,2,3$, ( having an even Grassmann character, the operator doesn't change the Grassmann character of spinors ) is recognised as the Dirac $\gamma^{m}$ matrices: $\tilde{\gamma}^{m} p_{m}|\tilde{\varphi}\rangle=0, m=0,1,2,3$. The third constraint of Eq.(3.1) has to be taken into account in the expectation value form $\left\langle\tilde{\varphi}\left|\tilde{\tilde{a}}^{m}\right| \tilde{\varphi}\right\rangle=0$.

In the presence of gauge fields the kinetic momentum $p_{m}$ in the Dirac equation has to be replaced by the canonical momentum: $p_{m} \longrightarrow p_{m 0}=p_{m}-A_{m}$, where $A^{m}$ stays for gauge fields 9, 10, 11]. 


\section{Poincaré group and Weyl-like equations for fermions and bosons.}

We are studying symmetries, connected with the transformation properties of ordinary and Grassmann coordinates. Besides the transformations of the Lorentz group $S O(1,3)$ in ordinary and Grassmann space, we allow translations in only ordinary space ${ }^{\text {** }}$. For the homogeneous transformations we have: $x^{\prime a}=L_{b}^{a} x^{b}+b^{b}, \theta^{\prime a}=L^{a}{ }_{b} \theta^{b}$, where $L^{a}{ }_{b}$ is defined in Sect. 2.1. and $a^{a}$ is any real constant vector. The operator, inducing these transformations, is then expressed as: $U(a, \omega)=e^{i a_{a} p^{a}-\frac{i}{2} \omega_{a b} M^{a b}}$. The operators of the infinitesimal translations in ordinary space $p^{a}$ and the Lorentz transformations in both spaces $M^{a b}$, which are according to Sects. 2. and 3. for spinors $M^{a b}=L^{a b}+\tilde{S}^{a b}$ and for vectors $M^{a b}=L^{a b}+S^{a b}$, close the (usual) Poincaré algebra [9]:

$$
\left\{p^{a}, p^{b}\right\}=0, \quad\left\{M^{a b}, p^{c}\right\}=i\left(\eta^{b c} p^{a}-\eta^{a c} p^{b}\right),
$$

with the commutator $\left\{M^{a b}, M^{c d}\right\}$ defined in Eq.(2.6).

Introducing the Pauli- Ljubanski vector $W^{a}=\left\{\frac{-3}{4 \eta} \Gamma, p^{a}\right\}$, where $\Gamma$ is defined in Eq.(2.7), one finds:

$$
W^{a} p_{a}=0, \quad\left\{p^{a}, W^{b}\right\}=0, \quad\left\{M^{a b}, W^{c}\right\}=i\left(\eta^{b c} W^{a}-\eta^{a c} W^{b}\right), \quad\left\{W^{a}, W^{b}\right\}=-i \epsilon^{a b}{ }_{c d} W^{c} p^{d},
$$

with $W^{0}=\vec{M} \cdot \vec{p}, \vec{W}=-\vec{M} p^{0}-\vec{K} \times \vec{p}$. The invariants of the Poincaré group are then:

$$
p^{2}=p^{a} p_{a}, \quad W^{2}=W^{a} W_{a}=M^{2} p^{2}-M_{a b} M^{c b} p^{a} p_{c} .
$$

For massless representations, which we only discuss in this paper, we have $p^{2}=0$.

\subsection{Little group and massless states.}

The representations of the Poincaré group can be obtained from the irreducible representations of the subgroup of the Poincaré group, called the little group [1, 3, 7], by the application of those generators of the Poincaré group which do not form the little group.

The little group is the set of all the Lorentz transformations which leave the four momentum of the state $k^{a}$ unchanged: $k^{\prime a}=L_{b}^{a} k^{b}=k^{a}$. For $L_{b}^{a}=\delta_{b}^{a}+\omega_{b}^{a}, \quad\left(\omega_{b}^{a}=\right.$ $\left.-\omega_{b}^{a}\right)$, it follows: $\omega_{b}^{a} k^{b}=0$.

If we choose $k^{a}=\left(k^{0}, 0,0, k^{3}\right), \quad k^{3} \geq 0, \quad$ then the requirement $\omega^{a}{ }_{b} k^{b}=0$ leaves free the three parameters of the little group: $\omega^{12}, \omega^{01}= \pm \omega^{13}, \omega^{02}=\mp \omega^{23}$, with the upper sign corresponding to $k^{0}=k^{3}$ and the lower sign to $k^{0}=-k^{3}$. This determines the three infinitesimal generators of the little group: $l^{1}:=M^{01} \pm M^{13}, l^{2}:=M^{02} \pm M^{23}, M^{12}$.

** Translations in Grassmann space cause 9] supersymmetric transformations, which shall be not studied here. 
They fulfil the commutation relations $\left\{l^{1}, l^{2}\right\}=0, \quad\left\{M^{12}, l^{1}\right\}=i l^{2}, \quad\left\{M^{12}, l^{2}\right\}=-i l^{1}$.

The invariant of the little group is $l^{2}=\left(l^{1}\right)^{2}+\left(l^{2}\right)^{2}$, while $W^{a}$, if applying on the little group representation, has the form $W^{a}=k^{3}\left(M^{12}, l^{2},-l^{1}, \pm M^{12}\right)$, where again the +1 is taken if $k^{0}=k^{3}$ and -1 if $k^{0}=-k^{3}$.

Paying attention on those representations which are eigenvectors of $l^{i}, i=1,2$, with the zero eigenvalue: $l^{1}=l^{2}=0$, only the rotations around the third axes are then nontrivially represented and form the $U(1)$ group. In this case, since $p^{2}=0=W^{2}$, it follows:

$$
W^{a}=\frac{W^{0}}{p^{0}} p^{a}=\frac{\vec{M} \cdot \vec{p}}{p^{0}} p^{a} .
$$

We easily find:

$$
\begin{aligned}
\left\{\frac{\vec{M} \cdot \vec{p}}{p^{0}}, p^{a}\right\}=0, \quad\left\{\frac{\vec{M} \cdot \vec{p}}{p^{0}}, M^{a b}\right\} & =\frac{i}{p^{0}}\left[\eta^{a 0}\left(W^{b}-\frac{W^{0}}{p^{0}} p^{b}\right)-\eta^{b 0}\left(W^{a}-\frac{W^{0}}{p^{0}} p^{a}\right)\right], \\
\left\{\frac{\vec{M} \cdot \vec{p}}{p^{0}}, \Gamma\right\} & =\frac{\vec{M} \cdot}{p^{0}}\left[\vec{W}-\vec{p} \frac{W^{0}}{p^{0}}\right] .
\end{aligned}
$$

According to Eq.(4.2b) the commutators $\left\{\frac{\vec{M} \cdot \vec{p}}{p^{0}}, M^{a b}\right\}$ and $\left\{\frac{\vec{M} \cdot \vec{p}}{p^{0}}, \Gamma\right\}$ vanish when acting in the representation space of the little group. The operator $\frac{\vec{M} \cdot \vec{p}}{p^{0}}$ commutes with all the generators of the Poincaré group and it is the invariant for the little group representations. The invariant of the Lorentz group $\Gamma\left(\left\{\Gamma, M^{a b}\right\}=0\right)$ is not the invariant of the Poincaré group, since $\left\{\frac{-3}{4 \eta} \Gamma, p^{a}\right\}=W^{a}$. However, $\vec{L} \cdot \vec{p}=0$ and accordingly $\frac{\vec{M} \cdot \vec{p}}{p^{0}}$ is equal to $\frac{\vec{S} \cdot \vec{p}}{p^{0}}$ for spinors and $\frac{\vec{S} \cdot \vec{p}}{p^{0}}$ for vectors, depending in both cases on only the spin and the direction of the momentum of the state. The operator $h:=\frac{\vec{M} \cdot \vec{p}}{|\vec{M} \vec{p}|}$ is called the helicity operator [6, 3]. Since $|\vec{p}|=\left|p^{0}\right|$ and $\{h, \Gamma\}=0$ on the little group representations, we can choose the little group representations to be eigenstates of not only the helicity operator $h$ but also of the internal part of the operator of handedness $\Gamma$ ( in Eq.(2.7) $M^{a b}$ arereplacedby $\tilde{S}^{a b}$, or $S^{a b}$, for the fermionic and bosonic case, respectively.) For the choice $k^{a}=\left(k^{0}, 0,0, k^{3}\right)$ the helicity operator is up to a constant equal to the operator $\tilde{S}^{12}$ for spinors and to the operator $S^{12}$ for vectors.

All the representations on Table I are eigenstates of either the operator $\tilde{S}^{12}$ or the operator of handedness $\tilde{\Gamma}$ and all the representations on Table II are eigenstates of either the operator $S^{12}$ or the operator of handedness $\Gamma$. To get the representations of the little group, the states on Table I and Table II have to be multiplied by the functions, which are the representation states of the momentum with the eigenvalue $k^{a}=\left(k^{0}, 0,0, k^{3}\right)$. 


\subsection{Weyl-like equations.}

If the eigenvalues of the operators of handedness $\Gamma$ and helicity $h=\frac{\vec{M} \cdot \vec{p}}{|\vec{M}||\vec{p}|}$ are nonzero on the little group representations, the eigenstates of these two operators fulfil the Weyl-like equation $((1.1))$

$$
\vec{M} \cdot \vec{p}=\xi \Gamma p^{0},
$$

with $M^{a b}=\tilde{S}^{a b}$ for spinors and $M^{a b}=S^{a b}$ for gauge three vectors with spin 1, with accordingly defined $\Gamma$ and with $\xi=\frac{1}{2}$ for spinors and 1 for vectors. While the two scalars from Table II trivially fulfil Eq.(4.3), the four vectors from Table II, having nonzero helicity and zero handedness, do not at all.

We easily see that on the little group representations the square of Eq.(4.3) leads to the equation $p^{2}=0$ for either the spinors or the three vectors. For spinors the square of Eq.(4.3) leads to the equation $p^{2}=0$ already on the operator level. In addition, if multiplying Eq.(4.3) in the spinor case by $\tilde{\Gamma}$, the Dirac equation for massless spinors $\tilde{\gamma}^{a} p_{a}=0$, presented in Sect. 3, follows.

Introducing the four vector $\mathcal{M}^{a}=(\xi \Gamma, \vec{M})$, again with $M^{a b}=\tilde{S}^{a b}, \xi=\frac{1}{2}$ for spinors and $M^{a b}=S^{a b}, \xi=1$ for vectors and for accordingly defined $\Gamma$, one can write

$$
\mathcal{M}^{a} p_{a}=0 .
$$

For the spinor states the operator equation (4.3a) can be written in the matrix form, if (for each of the four four vectors on Table I separately) the operators $\overrightarrow{\widetilde{S}}$ and $\tilde{\Gamma}$ are replaced by the four by four matrices, presented in Eq.(2.11a). Since the corresponding matrix equation has the block diagonal form, we may further replace it by the two Weyl equations for massless spinors: $\sigma^{a}{ }_{ \pm} p_{a}=0$, with $\sigma^{a}{ }_{ \pm}=( \pm I, \vec{\sigma})$, where $\vec{\sigma}$ are the Pauli matrices and $I$ is the two by two unit matrix, while $(+)$ staying for the left and $(-)$ for the right handed spinors, respectively.

One can proceed equivalently also for the gauge spin 1 vector states. The matrix form of the operator equation (4.3) follows, if the operators are replaced by six by six matrices from Eq.(2.11b). Since the matrix equation has the three by three block structure, we may further replace it by the two matrix equations: $s^{a}{ }_{ \pm} p_{a}=0$, with $s^{a}{ }_{ \pm}=( \pm I, \vec{s})$, for the left $(+)$ and the right $(-)$ handed vectors, respectively. Now $I$ is the unit three by three matrix and $\vec{s}$ are the three by three matrices, which define the adjoint representations of the group $S U(2)$ and are presented in Eq. (2.11b).

States, presented in Table I and Table II, are the spin part of the little group representations for the special case of momentum eigenvalues $k^{a}=\left(k^{0}, 0,0, k^{3}\right)$. For a general case $k^{a}=\left(k^{0}, \vec{k}\right)$, superpositions of states from Table I (for spinors) and Table II (for vectors) solve Eq.(4.3).

In the case of spinors the solutions of Eq.(4.3) for $k^{a}=\left(k^{0}, \vec{k}\right)$, expressed by the first four vectors from Table I ( one proceeds similarly also for the rest three cases) $\left\langle\left.\theta\right|^{\alpha} \tilde{\varphi}_{i}\right\rangle$, 
with $\alpha=1$ for left handed spinors and $\alpha=2$ for right handed spinors and $i=1,2$, is as follows:

$$
\left\langle x,\left.\theta\right|^{r} \tilde{\varphi}_{ \pm}\right\rangle=\mathcal{N}(x, k)\left\{\begin{array}{c}
\left\langle\left.\theta\right|^{\alpha} \tilde{\varphi}_{1}\right\rangle+\beta(k)\left\langle\left.\theta\right|^{\alpha} \tilde{\varphi}_{2}\right\rangle \quad, \quad \text { for } k^{0}=r|\vec{k}|, h=1 \\
-\beta^{*}(k)\left\langle\left.\theta\right|^{\alpha} \tilde{\varphi}_{1}\right\rangle+\left\langle\left.\theta\right|^{\alpha} \tilde{\varphi}_{2}\right\rangle \quad, \quad \text { for } k^{0}=-r|\vec{k}|, h=-1
\end{array}\right\}
$$

We choose $k^{3}=\left|k^{3}\right|$, while $\beta(k)=\frac{k^{1}+i k^{2}}{\left|k^{0}\right|+k^{3}}, \beta^{*}(k)$ means complex conjugate value to $\beta(k)$, $N(x, k)=\sqrt{\frac{k^{3}+\left|k^{0}\right|}{2\left|k^{0}\right|}}\langle x \mid k\rangle$ represents the normalization and the plane wave part. Solutions fulfil the condition $\left(k^{0}\right)^{2}=(\vec{k})^{2}$ and correspond to left handed spinors for $r=1$ and $\alpha=1$ and to right handed spinors for $r=-1$ and $\alpha=2$. The two helicity states have the index $+(h=1)$ and $-(h=-1)$.

In the case of the left and right handed three vectors from Table II, the solutions for a general case $k^{a}=\left(k^{0}, \vec{k}\right)$, for the left $(\alpha=1, r=1$,$) and for the right handed$ $(\alpha=2, r=-1)$ vectors with the helicities $h=1(+)$ and $h=-1(-)$ are as follows:

$$
\left\langle x,\left.\theta\right|^{r} \varphi_{ \pm}\right\rangle=\mathcal{N}(x, k)\left\{\begin{array}{c}
\left\langle\left.\theta\right|^{\alpha} \varphi_{1}\right\rangle+r \sqrt{2} \beta(k)\left\langle\left.\theta\right|^{\alpha} \varphi_{2}\right\rangle+(\beta(k))^{2}\left\langle\left.\theta\right|^{\alpha} \varphi_{3}\right\rangle, \\
\text { for } k^{0}=r|\vec{k}|, h=1 \\
\left(\beta^{*}(k)\right)^{2}\left\langle\left.\theta\right|^{\alpha} \varphi_{1}\right\rangle+r \sqrt{2} \beta^{*}(k)\left\langle\left.\theta\right|^{\alpha} \varphi_{2}\right\rangle+\left\langle\left.\theta\right|^{\alpha} \varphi_{3}\right\rangle, \\
\text { for } k^{0}=-r|\vec{k}|, h=-1
\end{array}\right\}
$$

The functions $\mathcal{N}(x, k), \beta(k)$ and $\beta^{*}(k)$ are the same as in the spinorial case. We again choose $k^{3}=\left|k^{3}\right|$ and assure that the condition $\left(k^{0}\right)^{2}=(\vec{k})^{2}$ is fulfilled. Again only two (rather than three) solutions exist for each handedness, one with helicity $h=+1$, the other with helicity $h=-1$. The former goes in the limit $\vec{k} \longrightarrow\left(0,0, k_{3}\right)$ to the vector with spin 1 , the latter to the vector with spin -1 .

The states of Eq.(4.4b) are one component wavefunctions with well defined handedness and helicity [3, 5, 4, 7]. To come from these one component objects to four component objects $A_{h r}^{a}$, which have well defined handedness $(r)$ and helicity $(h)$ and which fulfil the supplementary condition $p_{a} A_{h r}^{a}=0$, the one component wave functions from Eq.(4.4b) have to be multiplied by a part $e^{a}{ }_{h}(k)$ which depends on the helicity $(h)$, on $\operatorname{momentum}\left(k^{a}\right)$ and not on the handedness and which fulfils the condition $e_{h}^{a}(k) k_{a}=0$. We see that neither handedness nor helicity of states change with the Lorentz transformations. The orthonormalization condition $e_{h a}^{*}(k) e_{h^{\prime}}^{a}(k)=-\delta_{h h^{\prime}}$, where $(*)$ means complex conjugation, is imposed in addition. If one looks for the space like unit vectors $n_{i}, \quad i=1,2$, which both fulfil the orthonormalization equations $n_{1}{ }^{a} n_{2 a}=-\delta_{1,2}$, and $n_{i}{ }^{a} k_{a}=0$, one finds $e_{ \pm}^{a}(k)=\frac{1}{\sqrt{2}}\left( \pm n_{1}{ }^{a}-i n_{2}{ }^{a}\right)$, so that $A_{ \pm}^{a r}=e_{ \pm}^{a}(k)\left\langle x,\left.\theta\right|^{r} \varphi_{ \pm}\right\rangle$.

\footnotetext{
${ }^{\dagger \dagger}$ For all the four cases the odd $\alpha$ corresponds to the left handed spinor, the even $\alpha$ to the right handed spinor.
} 
One also remarks that the solution is not unique: for any chosen set $n_{i}{ }^{a}, i=1,2$, which solve our problem, the infinite set of vectors $n_{i}{ }^{a}+\lambda_{i} k^{a}, i=1,2$, where $\lambda_{i}$ are any function of $k^{2}$, is also a solution. This demonstrates the gauge invariance of the solutions $e_{h}^{a}(k)$ and correspondingly of $A_{h r}^{a}$.

\subsection{Discrete Lorentz symmetries.}

Starting from an initial vector one can find all vectors belonging to the same irreducible representation of the Lorentz group by applying the generators of the homogeneous Lorentz group. The generators of the extended Lorentz group, space and time inversion, bring us to other representations.

According to the fact that space has in our approach (the same number of) ordinary and Grassmann coordinates, the space and the time inversion in ordinary and Grassmann space occur. We define the space inversion operators for each of the two types of coordinates as follows:

$$
I_{s}^{x}\left(\begin{array}{c}
x^{a} \\
p^{a}
\end{array}\right)\left(I_{s}^{x}\right)^{-1}=\left(\begin{array}{c}
x_{a} \\
p_{a}
\end{array}\right) ; \quad I_{s}^{\theta}\left(\begin{array}{c}
\theta^{a} \\
p^{\theta a}
\end{array}\right)\left(I_{s}^{\theta}\right)^{-1}=\left(\begin{array}{c}
\theta_{a} \\
p_{a}^{\theta}
\end{array}\right),
$$

The space inversion operator for the hole space is accordingly the product of the above two operators: $I_{s}=I_{s}^{x} I_{s}^{\theta}$.

We define equivalently also the time inversion operators:

$$
I_{0}^{x}\left(\begin{array}{l}
x^{a} \\
p^{a}
\end{array}\right)\left(I_{0}^{x}\right)^{-1}=\left(\begin{array}{c}
-x_{a} \\
-p_{a}
\end{array}\right) ; \quad I_{s}^{\theta}\left(\begin{array}{c}
\theta^{a} \\
p^{\theta a}
\end{array}\right)\left(I_{s}^{\theta}\right)^{-1}=\left(\begin{array}{l}
-\theta_{a} \\
-p_{a}^{\theta}
\end{array}\right),
$$

The require that $p^{0}$, which means the energy, does not change sign under time inversion, can be fulfilled if the time inversion operator is antilinear. The time inversion operator for the whole space is therefore: $I_{0}=I_{o}^{x} I_{0}^{\theta} K$, where $K$ means complex conjugation. Accordingly it follows: $I_{0} x^{a}\left(I_{0}\right)^{-1}=-x_{a}, \quad I_{0} p^{a}\left(I_{0}\right)^{-1}=p_{a}, \quad I_{0} \theta^{a}\left(I_{0}\right)^{-1}=-\theta_{a}, \quad I_{0} p^{\theta a}\left(I_{0}\right)^{-1}=$ $p_{a}^{\theta}$.

We see by inspection of Table I and Table II that space inversion $I_{s}$ transforms left handed spinors into right handed ones and left handed vectors into right handed ones. Time inversion $I_{0}$ does not change handedness of states, either spinorial from Table I or vectorial of Table II. In the vectorial case we see that the time inversion transforms each of the two three vectors into themselves. The two scalars and the two four vectors of Table II have handedness equal to zero.

\section{Interacting massless fermions and gauge bosons.}

We discuss in this sections those properties of the interacting fields, which are connected with only spin degrees of freedom. We don't pay attention on charges. As long as the spin 
manifests as the internal degree of freedom under the application of the Lorentz group $S O(1,3)$, and the spin and the charge degrees of freedom are independent, states with different charges behave equivalently under the application of the generators of the Lorentz transformations. Using our approach, which describes the internal degrees of freedom of fields as dynamics in Grassmann space, we demonstrate that left handed spinors only interact with left handed gauge vectors and right handed spinors only interact with right handed gauge vectors, what is expected also from the group theoretical point of view.

According to what we said in Sect. 3., spinors always "see" coordinates $\theta^{a}$ proportional to their conjugate momenta $p^{\theta a}$. Because of that in the equations of motion for spinors all $\theta^{a}$ should be replaced by $\frac{1}{2} \tilde{a}^{a}$. The interacting term between spinors and gauge vectors follows, if in the equation for spinors $\overrightarrow{\vec{S}} \cdot \vec{p}=\frac{1}{2} \tilde{\Gamma} p^{0} \quad$ (Eq.(4.3)), the kinetic momentum $p_{a}$ is replaced by the canonical one: $p_{0 a}=p_{a}-A_{a r}$. Being interested in only handedness, we drop the helicity index. According to what we have said above, $A_{r}^{a}(\theta)$ has to be replaced by $A_{r}^{a}(\tilde{a})$, where the spin part of $A_{r}^{a}(\theta)$ is presented in Sects. 2.7 and 4.2. For the corresponding Lagrange term, describing the local interaction of a spinor and a gauge field, we accordingly have $\tilde{\mathcal{L}}_{\text {int }}=\left\langle{ }^{r} \tilde{\varphi} \mid x, \theta\right\rangle \tilde{S}_{a} A_{r^{\prime}}^{a}\left\langle x, \theta \mid r^{\prime \prime} \tilde{\varphi}\right\rangle$, where handedness $r, r^{\prime}, r^{\prime \prime}$ can take all possible values. Candidates for the spin part of spinor functions $\left\langle\left.\theta\right|^{r} \tilde{\varphi}\right\rangle$ are all functions from Table I, half of them left and half of them right handed, and for gauge vector functions $A_{r}^{a}$ are the two three vectors, one of the left and one of the right handedness from Table II.

In order to evaluate the $\tilde{\mathcal{L}}_{i n t}$, we first rewrite the two three vectors in terms of $\tilde{a}^{a}$. Then we find, if defining: $\tilde{S}^{ \pm}:=\tilde{S}^{23} \pm i \tilde{S}^{31}, \quad \tilde{K}^{ \pm}:=\tilde{S}^{01} \pm i \tilde{S}^{02}$, with $\tilde{S}^{a b}$ from Eq.(2.9) and if using the relation (Eq.2.5b) $\tilde{a}^{a} \tilde{a}^{b}=-2 i \tilde{S}^{a b}$, for $a \neq b$, the followig expressions

$\left\langle\left.\tilde{a}\right|^{1} \varphi_{1}\right\rangle=\frac{-1}{4}\left(\tilde{S}^{+}+i \tilde{K}^{+}\right), \quad\left\langle\left.\tilde{a}\right|^{1} \varphi_{2}\right\rangle=\frac{-\sqrt{2}}{4}\left(\tilde{S}^{3}+i \tilde{K}^{3}\right), \quad\left\langle\left.\tilde{a}\right|^{1} \varphi_{3}\right\rangle=\frac{1}{4}\left(\tilde{S}^{-}+i \tilde{K}^{-}\right)$, $\left\langle\left.\tilde{a}\right|^{2} \varphi_{1}\right\rangle=\frac{1}{4}\left(\tilde{S}^{+}-i \tilde{S}^{+}\right),\left\langle\left.\tilde{a}\right|^{2} \varphi_{2}\right\rangle=\frac{-\sqrt{2}}{4}\left(\tilde{S}^{3}-i \tilde{K}^{3}\right), \quad\left\langle\left.\tilde{a}\right|^{2} \varphi_{3}\right\rangle=\frac{-1}{4}\left(\tilde{S}^{-}-i \tilde{K}^{-}\right)$.

Taking these expressions for the left (the first three) and the right (the second three) handed three vectors into account, one easily checks with the help of Eq.(2.9) that only terms in which left handed spinors interact with left handed vectors and right handed spinors interact with right handed vectors give a nonzero contributions to the $\tilde{\mathcal{L}}_{\text {int }}$. Since the spinor states from Table I are all orthonormalized (Sects. 2.5 - 2.7.), integration over $\theta$ coordinates permits only terms with spinors of the same handedness.

The interacting part of the Lagrange density has accordingly the form:

$$
\tilde{\mathcal{L}}_{\text {int }}=\tilde{\varphi}_{L}^{+} \tilde{S}^{a} A_{a L} \tilde{\varphi}_{L}+\tilde{\varphi}_{R}^{+} \tilde{S}^{a} A_{a R} \tilde{\varphi}_{R} .
$$

with $\tilde{\varphi}_{L, R}=\left\langle x,\left.\theta\right|^{L, R} \tilde{\varphi}\right\rangle, \tilde{\varphi}_{L, R}^{+}=\left\langle{ }^{L, R} \tilde{\varphi} \mid x, \theta\right\rangle$, where $L, R$ stays for the left and the right handedness, respectively. Eq.(5.1) can also be understood from the group theoretical point of view: the group $S O(1,3)$, manifesting the $S U(2) \times S U(2)$ structure, determines left and right handed fundamental and left and right handed adjoint representations. To left (right) handed fundamental representations, the left (right) handed adjoint representations correspond. Accordingly, only a left (right) handed gauge boson can change spin 
of a left (right) handed fermion.

One can also find that the true scalar can not influence the spin part of spinor wave functions, while the pseudo scalar multiplies the left handed spinors by -1 and the right handed spinors by +1 . Accordingly the two superpositions of the two scalars from Table II $\frac{1}{2}\left(\left\langle\left.\tilde{a}\right|^{3} \varphi_{1}\right\rangle \pm\left\langle\left.\tilde{a}\right|^{4} \varphi_{1}\right\rangle\right)$ multiply spinors by 0 or 1 .

One can further check that the two four vectors from Table II, belonging to the representations $\left(\frac{1}{2}, \frac{1}{2}\right)$ and having $S^{2}=\frac{3}{2}$ (rather than 2), change handedness of spinors.

\section{Discussions and conclusions.}

Following the approach in which all the internal degrees of freedom of fields are determined within the Grassmann space, we found the Weyl-like equations not only for massless spinors but also for massless gauge vectors: $\vec{M} \cdot \vec{p}=\Gamma p^{0}$, where $\vec{M}$ and $\Gamma$ are operators which determine the spin and the handedness for either spinors or vectors, respectively ( $M^{a b}=\tilde{S}^{a b}$ for spinors and $M^{a b}=S^{a b}$ for vectors). Accordingly the eigenstates of

these two types of equations have well defined not only helicity $\left(h=\frac{\vec{M} \cdot \vec{p}}{|\vec{M} \| \vec{p}|}\right)$ but also handedness $(\Gamma)$. Massless vector fields demonstrate in addition the gauge invariance, as a consequence of the fact that two rather than three superpositions of basic states fulfil the condition $p^{2}=0$, for each of the two types of states. In the representation with well defined helicity and handedness are the operators, representing the generators of the Lorentz transformations and the handedness for the gauge vector fields the six times six matrices with three times three block diagonal structure ( which demonstrates the left and the right handedness, respectively), while for the spinor case they are four times four matrices with two times two diagonal structure ( again demonstrating the left and the right handedness, respectively). Among the vector representations we found also the true and the pseudo scalar of the zero handedness, trivially fulfilling the above equation. We further found, as expected, that the space reflection operator in Grassmann space transforms left handed fields into right handed ones and that the time inversion doesn't change the handedness of fields.

Looking at the interaction terms among spinors and gauge vectors we found that left handed spinors only interact with left handed gauge vectors, while right handed spinors only interact with right handed gauge vectors. The sum and the difference of the true scalar and the pseudoscalar manifest, when interacting with spinors, as projectors.

The experimentally measured gauge massless fields, the photon and the gluon, having well defined parity rather than handedness, are described by the sum of states of the left and the right handed three vectors, presented in this paper. Such states still have well defined helicity and still demonstrate the gauge invariance, but are no longer solutions of the Weyl-like equations. On the other side, the weak boson field, when interacting with fermions, only demonstrates its left handedness, while the quarks and the leptons 
manifest accordingly as left handed weak doublets.

Since handedness concerns the spin degrees of freedom, the answers to the questions why we only see photons and gluons with the positive parity and not also their partners with the negative parity, why weak bosons interact only with left handed fermions and why the left handed neutrino and antineutrino only are measurable, are to our understanding all connected. Also the answer to the question, how the break of left-right symmetry occurs, should depend on properties of photons, gluons, weak bosons and all fermions. Since fields of different charges demonstrate the handedness in different ways, only the unified approach to spins and charges can help to answer these questions.

In ref. [12, 13] the left-right symmetry of weak interaction is studied and it is made manifesting by the introduction of the two types of gauge $S U(2)$ fields, one of the left and the other of the right handedness. But handedness is the property of the Lorentz $S O(1,3)$ group, and has nothing to do with the weak charge, or any other charge, unless spins and charges are unified. In these models 12, 13 the left-right symmetry is broken through the Higgs mechanism with the two types of scalars, one of the left and one of the right handedness. But scalars have, as we showed, zero handedness.

The approach was proposed [9, 10, 11], in which all the internal degrees of freedom, spins and charges, are described in an unique way within the Grassmann space. It offers the way of understanding what is common to handedness and charges. Indeed, in a $S O(1,14)$ multiplet, demonstrating the subgroups $S O(1,3), S U(3), S U(2), U(1)$, the left handed $S U(2)$ doublets together with right handed $S U(2)$ singlets appear, while the right handed $S U(2)$ doublets appear together with the left handed singlets 16. This work is in progress.

We should comment at the end, what the four times two two component Weyl spinors could represent. We think that they represent the four (rather than three) families of quarks and leptons [9]. To show this a further study is needed.

\section{Acknowledgement.}

This work was supported by Ministry of Science and Technology of Slovenia. One of the authors would like to thank very much the theoretical group at the University of La Plata, where the part of this work was done, for the stimulating working atmosphere.

\section{References}

[1] E.P. Wigner, Ann. Math.149, 40 (1939),

[2] S.S.Schweber, An introduction to relativistic quantum field theory, Ed. Harper and Row publisher, New York (1962), 
[3] L. Fonda, G.C. Ghirardi, Theoretical physics vol.1., Symmetry principles in quantum physics, chapter 5, Marcel Dekker, New York, 1980,

[4] J.D. Bjorken, S.D. Drell, Relativistic quantum fields, chapter 14, Inernational series in pure and applied physics, McGraw-Hill book company 1965, New York at al,

[5] M. Gourdin, Basics of Lie groups, Editions frontiéres, Gif sur Yvette, 1982,

[6] S. Weinberg, Phys. Rev. 135 b, B1049 (1964),

[7] D. Han, Y.S. Kim, D. Son, Phys. Rev. D 26, 3713 (1982),

[8] R. Mirman, Massless representations of the Poincaré group, electromagnetism, gravitation, quantum mechanics, geometry, Nova scienca publisher, Inc., 1995,

[9] N. Mankoč Borštnik, J. Math. Phys. 34, 3731 (1993); J. Math. Phys. 36, 1593 (1995); N. Mankoč Borštnik, S. Fajfer, N. Cimento B, to appear,

[10] N. Mankoč Borštnik, Phys. Lett. B 292, 25 (1992); Nuovo Cimento A 105, 1461 (1992); IC/91/371; Int. Jour. Mod. Phys. A 9, 1731 (1994); Mod. Phys. Lett. A 10, 587 (1995); hep-th9408002; hep-th9406083; Proceedings of the International conference quantum systems, New trends and methods, Minsk, 23-29 May, 1994, p. 312, Ed. by A.O. Barut, I.D. Feranchuk, Ya.M. Shnir, L.M. Tomil'chik, World Scientific, Singapore 1995; Proceedings of the US-Polish Workshop physics from Plank scale to electroweak scale, Warsaw, 21-24 Sept. 1994, p. 86, Ed. by P. Nath, T. Taylor, S. Pokorski, World Scientific, Singapore 1995; Proceedings of the $7^{\text {th }}$ Adriatic meetings on High energy physics, Brijuni, Croatia, 13-22 Sept.1994, p. 296, Ed. D. Klabučar, I. Picek, D. Tadić, World Scientific, Singapore 1995; Proceedings of the Barut memorial conference on Group theory in physics, Tr. J. of Phys.21 321 (1997),

[11] N. Mankoč -Borštnik, Proceedings of the $V I I^{\text {th }}$ international conference Symmetry methods in physics, Dubna 10-18 July 1995, to appear,

[12] J.C. Pati, A. Salam, Phys. Rev. D 10, 275 (1974),

[13] R.N. Mohapatra, J.C. Pati, Phys. Rev. D 11, 566, 2558 (1975); G, Senjanovic̀, N. Mohapatra, Phys. Rev. D 12, 1502 (1975), R. N. Mohapatra, Unification and supersymmetry, The frontier of quark-lepton physics, Springer-Verlag (1986),

[14] F.A. Berezin and M.S. Marinov, The methods of second quantization, Pure and applied physics, Accademic press, New York, 1966,

[15] H. Ikemori, Phys.Lett. B 199, 239 (1987),

[16] N. Mankoč Borštnik, A. Borštnik, in preparation. 